\title{
Evaluation of Syndromic Surveillance for Opioid Overdose Reporting in Illinois
}

\author{
Frances Rose Lendacki ${ }^{1,2}$, Stacey Hoferka ${ }^{1}$ \\ ${ }^{1}$ Illinois Department of Public Health, Chicago, Illinois, United States, ${ }^{2}$ Division of Epidemiology \& Biostatistics, University of Illinois at Chicago, School \\ of Public Health, Chicago, Illinois, United States
}

\section{Objective}

To evaluate capacity of the BioSense ESSENCE platform and pre-defined overdose queries to identify emergency department admissions related to opioid overdose, in compliance with 2018 mandatory overdose reporting laws in Illinois

\section{Introduction}

Accuracy in identifying drug-related emergency department admissions is critical to understanding local burden of disease and assessing effectiveness of drug abuse prevention and overdose-reduction initiatives. In 2018 the Illinois Department of Public Health (IDPH) began implementation of a mandatory opioid overdose reporting law, applicable to all hospital emergency departments (ED). The mandate requires reporting of patient demographics, causal substance and antagonist ED administration within 48 hours of presentation. This reporting is not name-based. IDPH currently utilizes a near real-time syndromic surveillance (SyS) reporting system for all hospital ED, capturing most of the mandated criteria. Leveraging this existing system facilitates adherence to the mandate while imposing minimal additional burden of reporting on local hospitals. The Division of Patient Safety and Quality at IDPH has thus chosen to evaluate the completeness of overdose reporting and compliance with the opioid overdose mandate that have resulted from use of the current syndromic surveillance system.

\section{Methods}

Multi-level internal and external validation methods are being employed to evaluate the accuracy of opioid overdose reporting through syndromic surveillance. An initial internal evaluation compared overdoses captured using hospital discharge data (HDD) and SyS data. This analysis compared daily overdose counts in the two datasets from 166 Illinois facilities, from admissions from April 1 through June 30 2017, inclusive. The opioid overdose query from HDD referenced ICD-10 poisoning codes; SyS utilized the preset Opioid Overdose Version 1 (v1.0) query in the ESSENCE Tool from the CDC's National Syndromic Surveillance Program's BioSense Platform. Daily and quarterly overdose counts by surveillance method were compared and visualized by facility.

Three facilities were chosen for a secondary, case-level data comparison based on: magnitude of overdose discrepancies, overall overdose burden, and availability of linked data elements. Individual overdose visits were matched across SyS and HDD datasets based on: date of birth, sex and approximate date of admit. Cases identified in SyS but missing from (1) discharge diagnosis query and (2) discharge database overall were quantified. Cases identified by HDD that were (3) not identified in the SyS overdose query or (4) missing from the SyS database entirely were also counted.

\section{Results}

From April 1 to June 30 2017, among the 166 providers analyzed, the HDD query identified 2,998 opioid overdose-related visits; SyS identified 3,266 (268 cases or 8.9\% difference) (Figure 1, r=0.724). A total of $25(15 \%)$ of facilities had equivalent overdose visit counts between datasets: all were among those with low case burden (13 or fewer overdose visits per facility over the quarter). Among facilities with a higher number of overdose presentations, differences in quarterly case counts (SyS minus HDD) ranged from -56 to 120 . Discrepant counts were found in $85 \%$ of centers (Figure 2). HDD captured a larger number of overdoses in 93 facilities (56\%). SyS captured a larger number of overdoses in 48 facilities (29\%). The ten facilities with highest syndromic caseload accounted for $33 \%$ of overall case burden (1069); the ten with the highest discharge counts accounted for $29 \%$ (897 cases). However, the top ten facilities by surveillance type were notably different: the 2 nd and 3rd highest using syndromic surveillance ranked 30th and 41st using discharge surveillance over the same period. The center with 5th highest caseload by discharge criteria ranked 38th using syndromic surveillance. 
In secondary case-level analyses: across datasets from three facilities, both HDD and SyS captured $43.5 \%$ of overdoses, while $56 \%$ were only in SyS data and $0.5 \%$ were only in HDD. Discrepancies in the all-visit ("denominator") datasets were found, requiring follow-up with facilities directly.

\section{Conclusions}

Next steps in these evaluations include further characterization of cases missed differentially by syndromic and discharge surveillance. An external validation phase will engage facility staff to query the Electronic Medical Record directly. Hospital personnel will review and confirm opioid overdose events captured by SyS and hospital facilities will investigate and resolve discrepancies in data quality.

These analyses have the potential to inform more accurate definitions for opioid-related overdose seen in emergency departments. Such improved surveillance can aid allocation of medication (naloxone and naltrexone), promotion of intervention (i.e. methadoneassisted treatment programs), and drug abuse prevention. Engagement of facility staff in public health surveillance has resulted in 187 hospital-registered users for the BioSense Platform to date, demonstrating the ability of surveillance improvement efforts to foster public health partnership. Finally, optimizations of automated hospital surveillance systems can help reduce the burden of reporting overdoses and ED morbidity in general, to encourage time spent on monitoring and response.

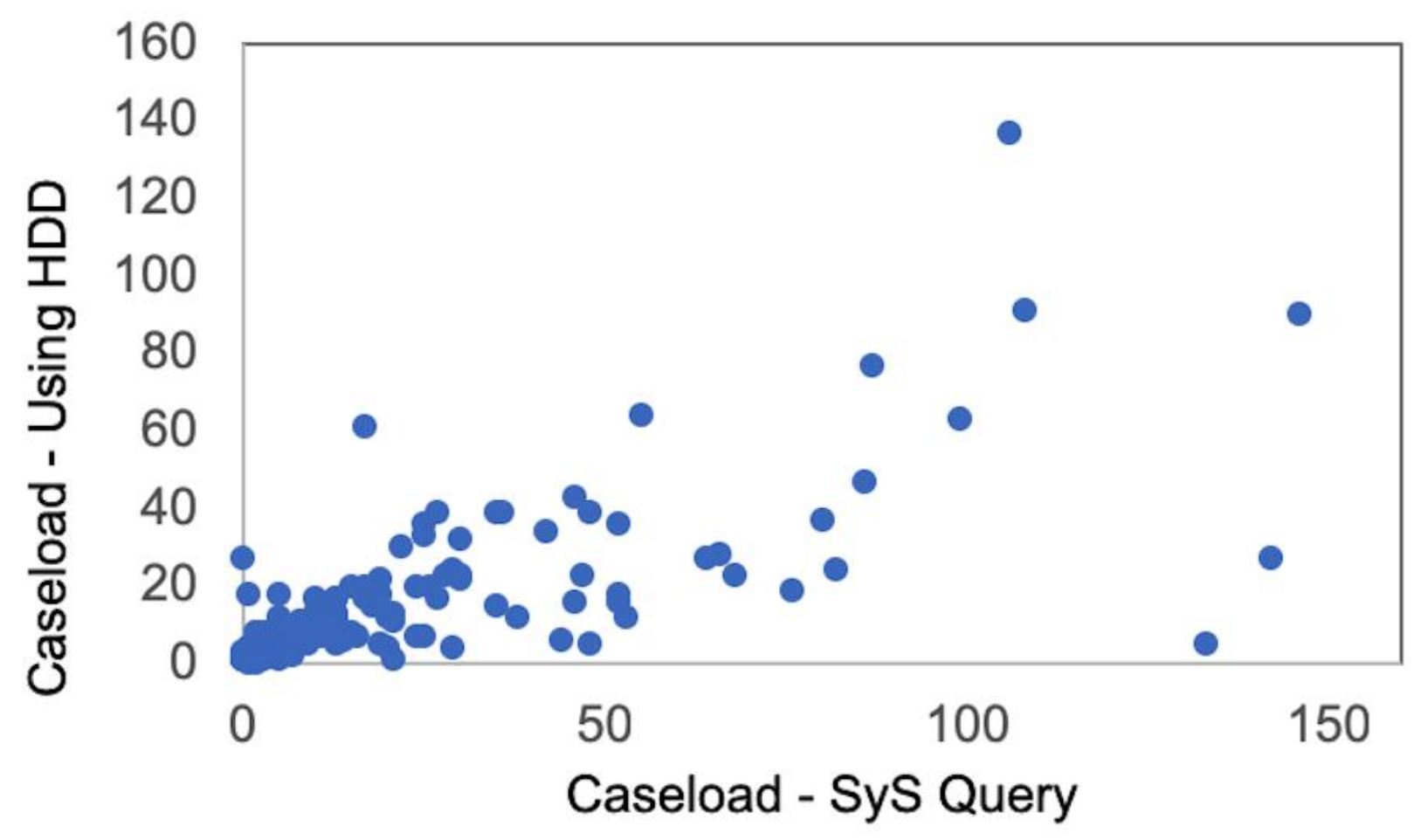

Figure 1. Correlation of Opioid Overdose Caseloads Derived from Syndromic Surveillance and Discharge Criteria in Illinois Emergency Departments, April - June 2017. n=166 facilities; Pearson correlation of quarterly caseloads by facility $=0.724$. 


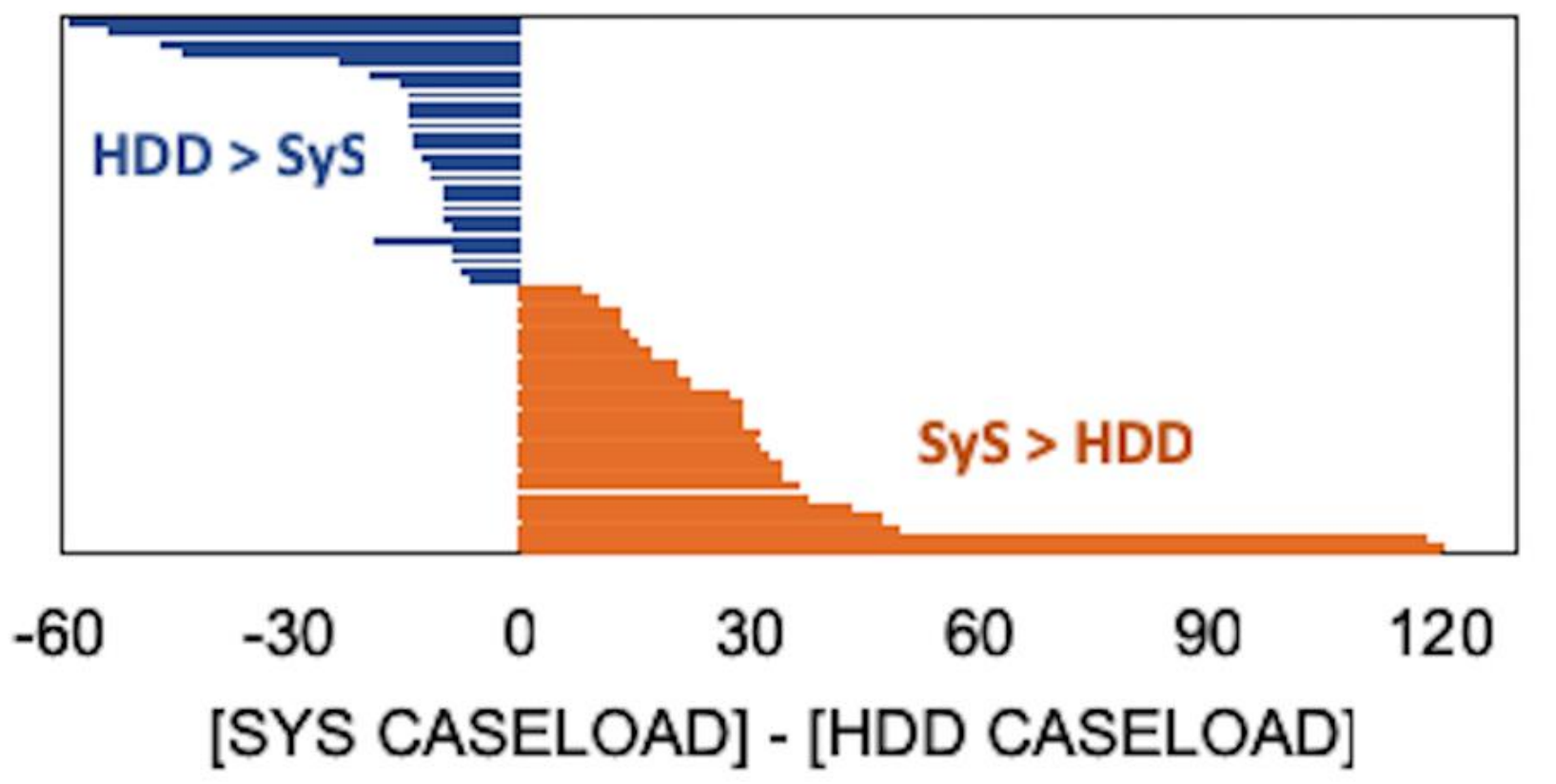

FIgure 2. Facility-level Discrepancies in Overall Opioid Overdose Counts Using Syndromic vs. Discharge Criteria in Illinois Emergency Departments, April - June 2017. Shown: of 166 facilities analyzed, 50 with greatest differences in aggregate quarterly counts of ED admissions related to opioid overdose, as identified using each surveillance method. 